Relations industrielles

Industrial Relations

\title{
Loi sur les relations de travail dans l'industrie de la construction
}

\section{Réjean Breton}

Volume 28, numéro 3, 1973

URI : https://id.erudit.org/iderudit/028425ar

DOI : https://doi.org/10.7202/028425ar

Aller au sommaire du numéro

Éditeur(s)

Département des relations industrielles de l'Université Laval

ISSN

0034-379X (imprimé)

1703-8138 (numérique)

Découvrir la revue

Citer cet article

Breton, R. (1973). Loi sur les relations de travail dans l'industrie de la construction. Relations industrielles / Industrial Relations, 28(3), 633-642.

https://doi.org/10.7202/028425ar

Tous droits réservés @ C Département des relations industrielles de l'Universite Laval, 1973
Ce document est protégé par la loi sur le droit d'auteur. L'utilisation des services d'Érudit (y compris la reproduction) est assujettie à sa politique d'utilisation que vous pouvez consulter en ligne.

https://apropos.erudit.org/fr/usagers/politique-dutilisation/ 


\title{
DROIT DU TRAVAIL
}

\section{LOI SUR LES RELATIONS DE TRAVAIL DANS L'INDUSTRIE DE LA CONSTRUCTION}

\section{Le nouvel article 33A autorise-t-il le maraudage permanent ?}

\author{
RÉJEAN BRETON
}

Le 8 juillet 1972, par la loi 58, le législateur créait la nouvelle centrale syndicale C.S.D. Par la même occasion, l'Assemblée nationale du Québec amendait la Loi sur les relations de travail dans l'industrie de la construction (Bill 290) en ajoutant l'article 33A qui est libellé de la façon suivante :

\begin{abstract}
«Aucun employeur, ni aucune personne agissant pour un employeur ou une association d'employeurs ne doit refuser d'employer ou licencier une personne parce que cette personne ou l'association de salariés dont elle est membre cesse ou déclare son intention de cesser d'être affiliée à une association représentative ou de payer des cotisations syndicales à telle association représentative. Aucune association représentative, ni aucune autre personne ne doit exercer des pressions de quelque façon que ce soit pour amener un employeur, une personne agissant pour un employeur ou une association d'employeurs à poser des actes visés par l'alinéa précédent.

Toute infraction au présent article rend le contrevenant passible des peines prévues à l'article $55 \mathrm{~A}$ 》.
\end{abstract}

Cet amendement est d'autant plus paradoxal que le législateur n'a pas jugé bon, à l'époque, de modifier l'article 33 qui stipulait ce qui suit :

-33. "Tout salarié a droit d'appartenir à une association de salariés de son choix et de participer à ses activités et à son administration. Il peut cependant entre le cent quatre-vingtième et le cent quarantième jour 1 précédant la date d'expiration du décret ou de la convention collective retirer son adhésion $\gg 2$.

\footnotetext{
* Breton, Réjean, professeur, Faculté de droit, Université Laval.
}

1 Cet article 33 a depuis été amendé par l'article 15 du Bill 9, sanctionné le 1er juin 1973, en remplaçant, dans la dernière phrase, les mots «entre le cent quatrevingtième et le cent quarantième jour 》 par les mots «pendant le septième mois 》. 
Comme il fallait s'y attendre, des travailleurs régis par le Décret de la construction se sont prévalus de ce nouvel article 33A pour révoquer l'autorisation écrite de retenue syndicale qu'ils avaient antérieurement remise à leur employeur (North Shore Builders Ltd.) et retirer également leur adhésion au syndicat auquel ils appartenaient jusqu'alors (Local 791, F.T.Q.). Parallèlement, ils signaient et remettaient à l'employeur une formule d'adhésion au syndicat des travailleurs de la construction, Beauce, Dorchester, Frontenac (C.S.D.) et l'autorisaient en même temps à prélever leurs cotisations syndicales et à les remettre au dernier syndicat nommé.

Évidemment, ces changements d'allégeance qui s'accompagnaient de retraits d'autorisation de retenues syndicales en faveur du syndicat affilié à la F.T.Q. sont survenus à des dates se situant à l'extérieur de la période de maraudage prévue au Décret de l'industrie de la construction en vigueur à l'époque.

Face à ces défections qui risquaient d'atteindre des proportions alarmantes, la F.T.Q. s'empressa de loger un grief selon la procédure prévue à l'article 13 du décret. Ce grief devait par la suite être référé à l'arbitre Jacques St-Laurent dont la décision ${ }^{3}$ fait l'objet du présent commentaire. Le procureur de la F.T.Q. fit valoir que ces changements d'appartenance syndicale étaient illégaux puisque survenus avant le début de la période de maraudage défini tant à l'article 33 du Bill 290 qu'à l'article 7.06, 1 - du décret qui s'appliquait alors.

L'arbitre saisi de l'affaire a rendu une décision dont les conclusions, si elles étaient retenues, risquent de perturber l'équilibre déjà précaire qui prévaut dans ce secteur effervescent qu'est celui de la construction.

Nous rendrons compte, dans un premier temps, des arguments qui fondent la sentence arbitrale rendue. Puis, dans un commentaire, nous tenterons de dégager quelle est l'interprétation qu'il faut donner des

2 Le Décret relatif à l'industrie de la construction dans la province de Québec (Arrêté en Conseil No 4599), qui expirait le 1er mai 1973, a repris cet article 33 ; il édictait, en son article 7.06,1 - : «Tout salarié ou membre peut, cependant, entre le 180 e et le $140 \mathrm{e}$ jour précédant la date d'expiration du décret, retirer son adhésion ou changer d'allégeance syndicale (dans le décret, à compter du 3 novembre 1972 jusqu'au 12 décembre 1972 inclusivement).

3 Cette décision arbitrale a été rendue le 1er juin 1973. 
articles 33 et 33A du Bill 290 de même que des articles 7 et 8 du décret qui expirait le 1er mai 1973.

\section{LA DÉCISION ARBITRALE}

L'arbitre ne retient pas les arguments du procureur de la F.T.Q. et rejette le grief en tenant les propos que voici.

Après avoir cité le nouvel article 33A du Bill 290, le professeur St-Laurent tient l'argumentation suivante :

«Aux yeux de l'arbitre, cet article est clair et donne le droit à un employé de cesser d'être affilié à une association représentative ou de payer des cotisations syndicales à telle association représentative. Selon cette loi, tous les employés de la construction, à partir du 8 juillet 1972, date à laquelle la loi 58 a été sanctionnée, avaient le droit de cesser d'appartenir à une association représentative et d'y verser des cotisations syndicales. Certains employés de l'employeur se sont prévalus de ce droit au cours du mois d'octobre 1972 aux dates mentionnées à l'annexe A. À partir de ces dates, et tant et aussi longtemps que les employés qui ont changé d'allégeance n'y changent rien, le local 191 (F.T.Q.) n'a aucun droit aux cotisations prélevées par l'employeur à l'égard de ces employés»(p. 4).

Le paragraphe suivant renferme la pierre angulaire de la décision arbitrale.

《Il est bien évident que la loi 58 qui, à la fois, créait par son article 3 la centrale syndicale démocratique (C.S.D.) et donnait par son article 4 le droit aux employés de la construction de cesser d'être affiliés aux deux seules associations représentatives à ce moment-là, soit la F.T.Q. et la C.S.N., et de ne plus payer des cotisations syndicales à ces associations représentatives, le faisait dans l'intention de permettre aux employés qui le désiraient d'adhérer à une centrale syndicale autre que la C.S.N. et la F.T.Q. Autrement, la loi 58 n'aurait pas de sens puisqu'elle ne permettrait alors que ce qui est déjà prévu au Décret de l'industrie de la construction. Or, une loi doit procurer un avantage. (Les italiques sont de nous). Il faut donc interpréter la loi comme accordant aux employés de la construction l'avantage de ne plus adhérer à l'une ou l'autre des deux (2) associations existantes et d'adhérer à la nouvelle association qu'elle créait». (pp. 4 et 5)

L'arbitre conclut là-dessus en affirmant que «la loi 58 accordait donc aux employés le droit de faire ce qu'ils ont fait ». Il précise encore :

《L'article 4 de la même loi (58) refusait même à l'employeur le droit de ne pas employer ou licencier une personne qui avait fait ce que ces employés ont fait ». (p. 5) 
Il faut ajouter qu'à ce stade de la sentence, le grief n'était pas encore complètement réglé. Restait toujours la question de savoir à qui devaient revenir les cotisations syndicales litigieuses que l'employeur avait jusque-là versées dans un compte « in trust » en attendant que le problème soit résolu. Devaient-elles être remboursées aux travailleurs concernés ou bien plutôt à la C.S.D. à laquelle ils avaient manifesté leur volonté d'adhérer?

L'arbitre est d'avis que ces cotisations doivent être remises aux travailleurs. Il s'explique de la façon suivante :

«Lorsque ces employés ont fait savoir à l'employeur qu'ils adhéraient à la C.S.D. et qu'ils l'autorisaient à retenir leur cotisation syndicale au profit de la C.S.D., ils le faisaient en conformité avec la loi 290.

«Ils le faisaient, évidemment, dans l'espoir que, conformément à l'article 6 de cette loi 290 , le ministre serait éventuellement dans l'obligation de reconnaître la C.S.D. comme association représentative ou que, sans y être obligé par l'article 6 de la loi 290, le ministre la reconnaisse quand même.

«Or, la C.S.D. n'a pas été reconnue comme association représentative. L'arbitre est donc d'opinion que la condition implicite d'adhésion à la C.S.D. n'ayant pas été réalisée, cette dernière n'a pas droit aux cotisations syndicales prélevées jusqu'ici...» (p. 5)

\section{COMMEnTAire}

Le grief soumis au professeur St-Laurent portait sur les défections de syndiqués survenues en dehors de la période de maraudage prévue, mais pendant la durée du décret. Ce n'est cependant pas une hypothèse d'école que d'envisager la survenance de cette situation non plus pendant la durée du décret, mais bien plutôt dans l'entre-temps des deux décerts.

Dans un cas comme dans l'autre, le litige soulève l'épineux problème de l'interprétation à donner des articles 33, 46 et 33A du Bill 290 et des articles 7 et 8 du Décret de la construction qui expirait le 1er mai 1973.

\section{Défections de syndiqués pendant la durée du décret}

Les défections qui ont occasionné le grief de la F.T.Q. sont survenues avant l'arrivée de la période de maraudage prévue à l'article 7.06,1 du décret, mais pendant la durée de ce dernier.

L'arbitre conclut que la loi 58 (plus spécifiquement le nouvel article 33A du Bill 290) accorde aux syndiqués le droit de faire ce qu'ils ont 
fait. Décider autrement, dit-il, ferait que cette loi 58 n'aurait plus aucune signification puisqu'elle ne permettrait que ce qui était déjà permis dans le Bill 290 et dans le décret avant qu'elle ne soit adoptée. Or, fait-il ressortir, se fondant sur l'article $41^{4}$ de la loi provinciale d'interprétation, «une loi doit procurer un avantage ».

Cette façon de voir de l'arbitre ne manque pas de poids, mais il faut bien reconnaitre qu'elle fait fi d'autres règles d'interprétation tout aussi importantes, sinon plus, que celle privilégiée dans sa décision.

"S'il est bien évident» que l'article 33A du Bill 290 est clair en lui-même, il n'en est plus ainsi si on l'envisage en tenant compte de l'article $46^{5}$ et surtout de l'article 33 déjà cité (de la même loi) que le législateur, il faut bien le souligner, n'a pas jugé opportun de faire disparaître. Si l'on tient compte encore des articles $7.01^{6}, 7.03^{7}, 7.06-1^{8}, 8.01^{9}$, et $8.02{ }^{10}$ du décret alors en application, l'on doit convenir que l'interprétation à donner de l'article $33 \mathrm{~A}$ perd beaucoup de sa limpidité.

4 «Toute disposition d'un statut, qu'elle soit impérative, prohibitive ou pénale, est réputée avoir pour objet de remédier à quelque abus ou procurer quelque avantage. Un tel statut reçoit une interprétation large, libérale, qui assure l'accomplissement de son objet et l'exécution de ses principes suivant leur véritable sens, esprit et fin ». Loi d'interprétation, S.R.Q. 1964, ch. 1.

5 Art. 46 « Tout employeur est tenu d'honorer l'autorisation écrite et irrévocable donnée par tout salarié de précompter chaque semaine sur son salaire le montant de la cotisation syndicale au bénéfice de l'association représentative indiquée...

Si l'employeur reçoit un avis de révocation dans un délai fixé à l'article 33, il doit en remettre une copie à l'association intéressée ».

6 Art. 7.01 - Adhésion obligatoire : «Tout salarié doit, comme condition de maintien de son emploi, devenir et demeurer membre en règle de l'une ou l'autre des associations syndicales représentatives de son choix...»

7 Art. 7.03 - Maintien d'affiliation: «Tout membre de l'une ou l'autre des associations syndicales représentatives doit maintenir son adhésion à l'association syndicale représentative dont il est membre pour la durée du décret, sous réserve du paragraphe 7.06-1》.

8 Art. 7.06-1 - Voir supra, note (2).

9 Art. 8.01-1 - Autorisation: «L'employeur doit exiger de tout salarié à son emploi une autorisation écrite et irrévocable de déduire le montant de la cotisation syndicale du syndicat ou de l'union auquel ou à laquelle il appartient».

10 Art. 8.02-4 - Obligation de l'employeur : «L'employeur qui reçoit un avis de révocation dans les délais fixés à l'article 33 de la Loi sur les relations de travail dans l'industrie de la construction, chapitre 45 des lois de 1968 et amendements doit en remettre copie à l'association syndicale représentative intéressée ». 
En effet, l'interprétation retenue par le professeur St-Laurent en est une qui isole le nouvel article 33A de son contexte ; ce dernier comprend surtout l'article 33 et les articles du Décret de la construction déjà cités. Puisque le législateur, en ajoutant 33A au Bill 290, a laissé intact l'article 33, il faut donc présumer qu'il avait des raisons d'agir ainsi et surtout, pour retourner contre l'arbitre la base même de son argumentation, qu'il (le législateur) entendait, en maintenant l'article 33, faire en sorte que ce dernier « remédie à quelque abus ou procure quelque avantage »11.

Dès lors que $33 \mathrm{~A}$ et 33 semblent aller en sens inverse, et comme le législateur n'est pas censé se contredire, il faut donc interpréter chacun de ces deux articles à la lumière de l'autre et de leur contexte et non, comme l'a fait l'arbitre, ignorer l'un pour privilégier l'autre. Il apparaît alors que l'intention du législateur, en édictant $33 \mathrm{~A}$ et en maintenant 33, est d'empêcher que la venue de la nouvelle centrale syndicale C.S.D. ne soit courtcircuiter, pendant la période de maraudage (art. 33), par des manoeuvres de représailles pour changement d'allégeance en sa faveur. Autrement, si l'on adopte l'interprétation retenue par le professeur, qui autorise les travailleurs de la construction à se retirer de leur syndicat et à cesser de leur verser les cotisations à partir de l'entrée en vigueur ( 8 juillet 1972) de la loi 58 amendant le Bill 290 et ce, en tout temps (même en dehors de la période de maraudage prévue à l'article 33), cela nous conduit à conclure que le législateur se contredit en ce qu'il édicte quelque chose à l'article 33 dont il s'empresse d'annuler les effets à l'article 33A.

Nous ajouterons encore que le contexte de l'article 33A comprend, outre l'article 33, plusieurs articles du Décret de la construction dont les plus pertinents ont déjà été cités. Toutes ces prescriptions sous-tendent une pratique des relations de travail qui prévaut depuis plusieurs années. Est-il permis de prétendre que le législateur a entendu faire disparaître tout cela en adoptant une mesure (article 33A) dont l'objet ne serait somme toute qu'implicite (puisqu'il maintient l'article 33)?

Si tous ces arguments ne suffisaient pas, il serait encore possible d'invoquer les conséquences particulièrement inquiétantes qu'entraînerait l'adoption de la position du professeur St-Laurent. Celle-ci conduirait à instaurer le maraudage comme état permanent. L'on connaît les trop

11 Article 41 de la Loi provinciale d'interprétation déjà cité, voir supra, note note (4). 
célèbres abus auxquels donne lieu la période (auparavant 40 jours, maintenant 1 mois, et ce, depuis le 1er juin 1973) de maraudage prévue à l'aticle 33. Voilà pourquoi si, à la limite, les deux interprétations de l'article $33 \mathrm{~A}$ étaient également plausibles, comme l'on peut présumer que la paix industrielle demeure une préoccupation majeure du législateur, il faudrait donc pencher du côté d'une solution qui tienne compte de cette préoccupation.

Une autre conséquence pour le moins troublante de cette sentence arbitrale réside dans l'éventuelle remise en cause de la survie même du syndicalisme dans ce secteur. En effet, si les travailleurs de la construction peuvent, en tout temps, retirer leur adhésion à un syndicat et cesser d'y verser leurs cotisations syndicales, cette même interprétation de l'article 33A nous autoriserait à penser, en continuant toujours d'ignorer les articles 33 et 46 du Bill 290 de même que les articles pertinents du décret, que ces travailleurs pourraient également s'abstenir de toute appartenance syndicale et de tout versement des cotisations qui s'y rattachent ${ }^{12}$.

\section{Défections de syndiqués dans l'entre-temps de deux (2) décrets}

Il ne nous semble pas inopportun d'élargir maintenant le débat pour envisager quelle serait la solution à donner à un litige consécutif à des défections syndicales survenues non plus pendant la durée d'un décret, mais après son expiration et avant que le suivant n'entre en vigueur.

La Loi sur les relations du travail dans l'industrie de la construction ne nous éclaire pas beaucoup si on adopte l'interprétation des articles 33 et $33 \mathrm{~A}$ que nous avons donnée dans la première partie de notre commentaire. Quant au décret, son article $7.03{ }^{13}$ pourrait peut-être constituer un élément de solution puisqu'il contient cette précision : «pour la durée du décret ». Est-ce à dire, a contrario, qu'un membre pourrait toujours retirer son adhésion à l'association syndicale représentative dans l'entretemps de deux (2) décerts ? Si c'était le cas, comment expliquer que les travailleurs de la construction ne se soient pas prévalus de ce droit depuis le 1 er mai 1973 ?

12 Concernant la propriété des cotisations syndicales litigieuses, l'arbitre décide, comme nous l'avons déjà dit, qu'elles doivent être remboursées aux travailleurs concernés, et non versées à la C.S.D., à moins que celle-ci ne soit consacrée association représentative par le Ministre du Travail.

13 Voir supra, note (7). 
L'article $61 \mathrm{du}$ Code du travail pourrait également nous fournir un indice de solution. Évidemment, l'article 59 du Bill 290 stipule que: «Les dispositions du Code du travail et de la Loi des décrets de convention collective ne s'appliquent pas dans l'industrie de la construction, sauf au cas de mention expresse», ce qui n'est pas le cas pour l'article 61, mais, si cet article ne s'applique pas comme tel, il n'en demeure pas moins que ce sont des préoccupations similaires qui ont présidé à l'élaboration de l'article 61 c.t. et des articles 33 et 33A du Bill 290. En effet, le législateur entendait, aussi bien pendant la durée d'une convention collective que d'un décret, instituer une période de trève. La prohibition de toute grève et de tout lock-out (article 23 du Bill 290 et aticles 95 et $97 \mathrm{du}$ Code du travail) en constitue la manifestation principale.

Cet article 61 c.t. est interprété comme signifiant que la prohibition qu'il édicte ne joue pas en l'absence d'une convention collective ${ }^{14}$. Est-ce à dire, par analogie, qu'il serait toujours permis de faire du maraudage dans l'entre-temps de deux (2) décrets? On pourrait être tenté de le croire, d'autant plus que les transferts d'affiliation individuelle ne sont pas défendus par l'article 61 c.t.

En dépit de ces deux arguments fondés sur des textes, nous ne pensons pas que cette solution doive être retenue. Nous fondons notre position sur le modèle de relations de travail foncièrement original qui prévaut dans le secteur de la construction.

Dans les relations de travail régies par le Code du travail, le syndicat accrédité détient le monopole de représentation des salariés face à l'employeur. Puisqu'il détient, seul, cette position privilégiée, pendant toute la durée de la convention collective (sauf pour les courtes périodes prévues à l'article $21 \mathrm{~d}$ ) et à l'article 61 du Code du travail), il est normal que ses rivaux puissent faire des démarches (art. 61 c.t.) dans l'entretemps de deux (2) conventions collectives, qu'ils puissent se manifester jusqu'à un certain point.

Par contre, dans le secteur de la construction, le pluralisme syndical joue d'une façon permanente. Pendant la durée du décret toutes les associations représentatives peuvent participer à son administration, de même qu'elles peuvent collaborer à son élaboration. Dès lors, ce modèle diffé-

14 Denis Gagnon plaignant et la Centrale des Syndicats démocratiques inculpée, (1973) T.T. 1. 
rent de relations de travail commande une solution différente face aux défections syndicales survenant en contexte de vide juridique. Il semble alors que les «partenaires» syndicaux se retrouvent sensiblement dans la même situation que pendant la durée du décret, puisque, étant représentatifs, ils demeurent toujours présents aux relations de travail. En d'autres termes, aucune association représentative ne jouissant d'un statut privilégié pendant la durée du décret (contrairement à l'association de salariés accréditée pendant la convention collective), il n'est donc plus nécessaire de pallier à ce déséquilibre en préconisant le maraudage pour toute la durée de l'entre-temps de deux (2) décrets.

Du reste, la pratique qui prévaut dans le secteur de la construction (abstraction faite des défections engendrées par le Bill 58) est à l'effet de limiter le maraudage à la période définie à l'article 33. C'est là un indice pour le moins sérieux de l'intention du législateur en cette matière.

Mais alors, n'est-il pas d'autant plus impérieux que les textes soient clarifiés en conformité avec cette pratique bien établie? Trop souvent l'on se plaint des difficultés qu'engendrerait une intervention législative dans ce secteur complexe de la construction ; pour une fois que les centrales syndicales s'entendent sur un «modus vivendi », il serait trop bête que les carences d'un texte législatif ne favorisent des écarts à cette entente.

\section{CONCLUSION}

La décision de l'arbitre St-Laurent ne termine pas définitivement cette affaire d'affiliation et de cotisations syndicales. Cette sentence a déjà fait l'objet d'une requête pour l'émission d'un bref d'évocation.

Dès à présent, il est permis de s'interroger sur les raisons qui ont poussé le législateur à édicter ce nouvel article 33A tout en maintenant l'article 33. On peut se demander s'il ne s'agit pas, encore là, davantage d'une manoeuvre politique que d'une oeuvre législative, visant à faire plaisir à tout le monde alors que, foncièrement, non seulement cette mesure n'apporte-t-elle aucune amélioration, mais encore elle véhicule des difficultés nouvelles. On maintient l'article 33 intact, et ce faisant, on laisse croire aux associations représentatives en place que les règles du 
jeu demeurent inchangées malgré l'entrée en scène de la nouvelle venue (C.S.D.) ; d'autre part, l'on ajoute ce paradoxal article 33A qui constitue, à n'en pas douter, une flagrante invitation au maraudage en tout temps, et alors, on ne saurait de prime abord déplaire outre mesure à la nouvelle centrale syndicale. L'importation de cet art bien connu du clair-obscur en matière législative ne nous semble pas la voie la mieux choisie par notre Assemblée nationale pour atteindre à la célébrité, et surtout pas aux yeux de ceux qui sont appelés à rendre des décisions en application de textes de cette nature.

La coexistence des articles 33 et $33 \mathrm{~A}$ constitue une autre illustration de ce procédé qui consiste, de la part du législateur, à abdiquer ses responsabilités en faveur (!) des juges et arbitres à qui, trop souvent, l'on demande de faire preuve d'une puissante imagination créatrice.

\title{
UNIQUE - INDISPENSABLE - UNIQUE - INDISPENSABLE \\ VOCABULAIRE FRANÇAIS-ANGLAIS DES RELATIONS PROFESSIONNELLES
}

\author{
GLOSSARY OF TERMS \\ USED IN \\ INDUSTRIAL RELATIONS \\ (ENGLISH-FRENCH) \\ Gérard DION \\ département des relations industrielles \\ Université Laval
}

Ouvrage comprenant plus de 4,000 mots ou expressions avec leur équivalent anglais. De même aussi une liste des sigles pour désigner les diverses organisations dans ce domaine.

A reference work including more than 4,000 terms with their French equivalents and a list of abbreviations (French and English) used to designate organizations in this field.

Un volume $6 \times 9$ relié 304 pages

$A$ hard-cover book $6 \times 9304$ pages

Prix - Price $\$ 10.00$

LES PRESSES DE L'UNIVERSITÉ LAVAL

C.P. 2447, QUÉBEC 2, (QUÉ.)

UNIQUE - INDISPENSABLE - UNIQUE - INDISPENSABLE 